\title{
Effects of Planting Date and Growth Hormone on Growth and Yield of Cauliflower
}

\author{
M. A. Rahman ${ }^{1}$, M. Imran ${ }^{2 *}$, M. Ikrum ${ }^{3}$, M. H. Rahman ${ }^{1}$ and M. G. Rabbani ${ }^{1}$ \\ ${ }^{1}$ Department of Horticulture, Bangladesh Agricultural University, Mymensingh-2202 \\ ${ }^{2}$ Hokakido University, Japan \\ ${ }^{3}$ ACI, Bangladesh \\ *Corresponding author: imran.pstu@gmail.com
}

\begin{abstract}
An experiment was conducted at the Horticulture Farm of Bangladesh Agricultural University, Mymensingh, during the period from October 2014 to March 2015 to study the effects of planting date and growth hormone on the growth and yield of cauliflower. The experiment consisted of two factors; Factor A: Three planting dates, such as $\mathrm{P}_{1}$ : Planting on 1 November; $\mathrm{P}_{2}$ : Planting on 15 November and $\mathrm{P}_{3}$ : Planting on 1 December; Factor B: Four levels of Growth hormone, such as $\mathrm{H}_{0}$ : No Hormone (control); $\mathrm{H}_{1}$ : $10 \mathrm{ppm}$ IAA (Indole-3 Acetic Acid); $\mathrm{H}_{2}: 70 \mathrm{ppm} \mathrm{GA}$ (Gibberellic Acid) and $\mathrm{H}_{3}: 10 \mathrm{ppm} \mathrm{IAA}+70 \mathrm{ppm} \mathrm{GA}$. The experiment was laid out following Randomized Complete Block Design (RCBD) with three replications. In case of planting date, the highest plant height $(63.26 \mathrm{~cm})$, number of leaves per plant $(24.13)$, leaf length $(59.26 \mathrm{~cm})$, leaf breadth $(19.31 \mathrm{~cm})$ at harvest, curd diameter $(22.25 \mathrm{~cm})$, marketable yield per hectare $\left(28.11 \mathrm{t} \mathrm{ha}^{-1}\right)$ were recorded from $\mathrm{P}_{2}$ and the lowest of those parameters were recorded from $\mathrm{P}_{3}$. In case of growth hormone the highest plant height $(63.10 \mathrm{~cm})$, number of leaves per plant $(23.66)$, leaf length $(59.05 \mathrm{~cm})$, leaf breadth $(18.98$ $\mathrm{cm})$ at harvest, curd diameter $(22.39 \mathrm{~cm})$, marketable yield per hectare $\left(29.88 \mathrm{t} \mathrm{ha}^{-1}\right)$ were recorded from $\mathrm{H}_{3}$ and the lowest of those perameters were recorded from $\mathrm{H}_{0}$. Combination of planting date and growth hormone the highest plant height $(65.96 \mathrm{~cm})$, number of leaves per plant $(26.42)$, leaf length $(63.64 \mathrm{~cm})$, leaf breadth $(20.92 \mathrm{~cm})$ at harvest, curd diameter $(25.75 \mathrm{~cm})$, marketable yield per hectare $\left(31.03 \mathrm{t} \mathrm{ha}^{-1}\right)$ were recorded from planting on 15 November and $10 \mathrm{ppm}$ IAA with $70 \mathrm{ppm} \mathrm{GA}_{3}\left(\mathrm{P}_{2} \mathrm{H}_{3}\right)$ and the lowest parameters $\left(21.75 \mathrm{t} \mathrm{ha}^{-1}\right)$ were recorded from planting on 1 December and no hormone $\left(\mathrm{P}_{3} \mathrm{H}_{0}\right)$. It is apparent from the above results that the combination of planting on 15 November and $10 \mathrm{ppm}$ IAA with $70 \mathrm{ppm} \mathrm{GA}_{3}\left(\mathrm{P}_{2} \mathrm{H}_{3}\right)$ was more productive from the other combinations.
\end{abstract}

Key Words: Cauliflower, Growth hormone, Planting date and Yield

\section{Introduction}

Cauliflower (Brassica oleracea var. botrytis sub var. cauliflora) is one of the most important cole crops belonging to the family Brassicaceae. The leading cauliflower production countries of the world are China, Pakistan and India in respect of yield per hectare of land. Cauliflower is a very tasty and much popular vegetable in Bangladesh as well as all over the world. $100 \mathrm{~g}$ edible part of cauliflower contains $89 \%$ moisture, 8.0 g carbohydrates, 2.3 g proteins, 40 IU carotene, 0.13 $\mathrm{mg} \mathrm{B} \mathrm{B}_{1}, 0.11 \mathrm{mg} \mathrm{B} \mathrm{B}_{2}, 50 \mathrm{mg}$ vitamin $\mathrm{C}, 30 \mathrm{mg}$ calcium and $0.8 \mathrm{mg}$ iron and also contains 30 calories (Rashid, 1999). Edible part of cauliflower is commonly known as 'Curd'.

Vegetable consumption in Bangladesh is very low, only $32 \mathrm{~g}$ per person per day against the minimum recommended quantity of $200 \mathrm{~g}$ per day (FAO, 2007). The total vegetable production in Bangladesh is far below the requirement. In 2012- 2013 cauliflower covered an area of 30,900 hectares with a total production of 101,485 metric tones (BBS, 2012). The suitable temperature for growth stages ranging from $20^{\circ}$ $\pm 5^{\circ} \mathrm{C}$. The best temperature for curd growth and development is $15^{\circ} \mathrm{C}$ to $20^{\circ} \mathrm{C}$. Planting date is one of the important factors for successful production of cauliflower. As a seasonal crop, cauliflower needs to plant in optimum time to return the maximum yield and benefit. Early planting produced the largest curd and maximum yield compared to the late planting. Malformation of curd also been reported in case of too early planting and late planting reduces curd size (Katharine, 1963). Many experiments have been carried out in developed country to investigate the effect of biochemical substances on the yield and quality of cauliflower. Reports so far been made indicated a promising results on yield and quality of cauliflower and other crops due to the use of bio-chemical substances, such as Napthaline acetic acid (NAA), Gibberellic acid $\left(\mathrm{GA}_{3}\right)$, Indole acetic acid (IAA) etc. (Voronova and Kozakov, 1983; Senthelhas et al., 1987; Tadzhiryan, 1990; Tomar et al., 1991). In addition, it is generally accepted that a biochemical processes are affected by a single chemical or a mixture of chemicals is not only different for between species but also for cultivars within the species and due to climatic regions (Hardy, 1979). However, recently done preliminary trials indicate possibility of yields increase of Cauliflower in Bangladesh with the use of biochemical (Islam el al., 1993; Biswas and Mondal, 1994). Plant height, curd formation and curd size of Cauliflower can be increased with foliar application of plant growth hormone. Several experiments were conducted to increase the yield of cauliflower. $\mathrm{GA}_{3}$ and IAA have a positive role on curd formation and curd size of cauliflower (Sharma and Mishra, 1989).

At present growth hormones are widely used in horticultural crop production all over the world. Growth hormones play a vital role in growth and development of cauliflower. Some plant growth hormones like Auxin, Gibberellins, Cytokinin etc. are involved with the physiological activities in plants. IAA is one of the plant growth regulators (PGRs) play an important role in cell division, promoting cell elongation, callus formation which enhance plants vegetative growth. Gibberellins are also important PGR, control plant growth and development with the most interesting with respect to the photoperiodic control of flowering. The cauliflower plant showed that $\mathrm{GA}_{3}$ at $100 \mathrm{ppm}$ 
produced the tallest plants, the largest curds and highest curd yields (Vijay and Ray, 2000).

Considering the background stated above, the present study was undertaken to investigate the effect of planting date and growth hormone with the following objectives-

i. to find out the appropriate planting date of cauliflower in relation to yield contributing characters and yield;

ii. to find out the appropriate amount of growth hormone of cauliflower in relation to yield contributing characters and yield; and

iii. to know the combined effect of planting date and growth hormone for ensuring the maximum growth and higher yield of cauliflower.

\section{Materials and Methods}

The present investigation was carried out at the Horticulture Farm, Bangladesh Agricultural University, Mymensingh, during the period from October 2014 to March 2015.The soil of the experimental plot was silty loam in texture belonging to the Old Brahmaptura Flood Plain. The experimental area was under the subtropical monsoon climate, characterized by heavy rainfall during Kharif season (April to September) and scanty rainfall in Rabi season (October to March). The collected seedlings were raised at the Horticulture Farm, Bangladesh Agricultural University, Mymensingh. The size of seedbed was $3 \mathrm{~m} \times 1 \mathrm{~m}$. The experiment was undertaken to study the effects of three different levels of planting dates and four different levels of growth hormones on the growth and yield performance of cauliflower. The experiment included two factors as follows:

Factor A: Planting dates - Three dates

i. $\mathrm{P}_{1}$ : Planting on 1 November

ii. $\mathrm{P}_{2}$ : Planting on 15 November

iii. $\mathrm{P}_{3}$ : Planting on 1 December

Factor B: Growth hormones - Four levels

i. $\mathrm{H}_{0}$ : No Hormone

ii. $\mathrm{H}_{1}: 10$ ppm IAA (Indole-3 Acetic Acid)

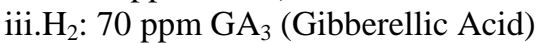

iv. $\mathrm{H}_{3}: 10 \mathrm{ppm} \mathrm{IAA}+70$ ppm GA 3

Thus there were 12 treatment combinations.

\section{Results and Discussion}

The experiment was considered of three of planting dates $(\mathrm{P})$ and four levels of growth hormones $(\mathrm{H})$ and was designed to find out the individual and combined effects on the growth and yield of cauliflower. The results have been presented and discussed and possible interpretations are given under the following headings:

\section{Plant height}

At 30, 40, 50, 60 DAT and at harvest the tallest plant 26.73, 42.67, 52.55, 60.74 and $63.26 \mathrm{~cm}$ was recorded from $\mathrm{P}_{2}$ (planting at 15 November) and the shortest plant 24.94, 37.59, 47.31, 52.26 and $53.12 \mathrm{~cm}$ was observed from $\mathrm{P}_{3}$ (planting on 1 December) at 30, 40, 50, 60 DAT and at harvest, respectively (Fig. 1).

At 30, 40, 50, 60 DAT and at harvest the tallest plant $27.1,42.67,53.12,61.94$ and $63.10 \mathrm{~cm}$ was found from $\mathrm{H}_{3}(10 \mathrm{ppm}$ IAA $+70 \mathrm{ppm} \mathrm{GA}$ ) and the shortest plant $24.08,37.23,45.49,50.07$ and $52.38 \mathrm{~cm}$ was obtained from $\mathrm{Ho}$ (no hormone) respectively (Fig. 2).

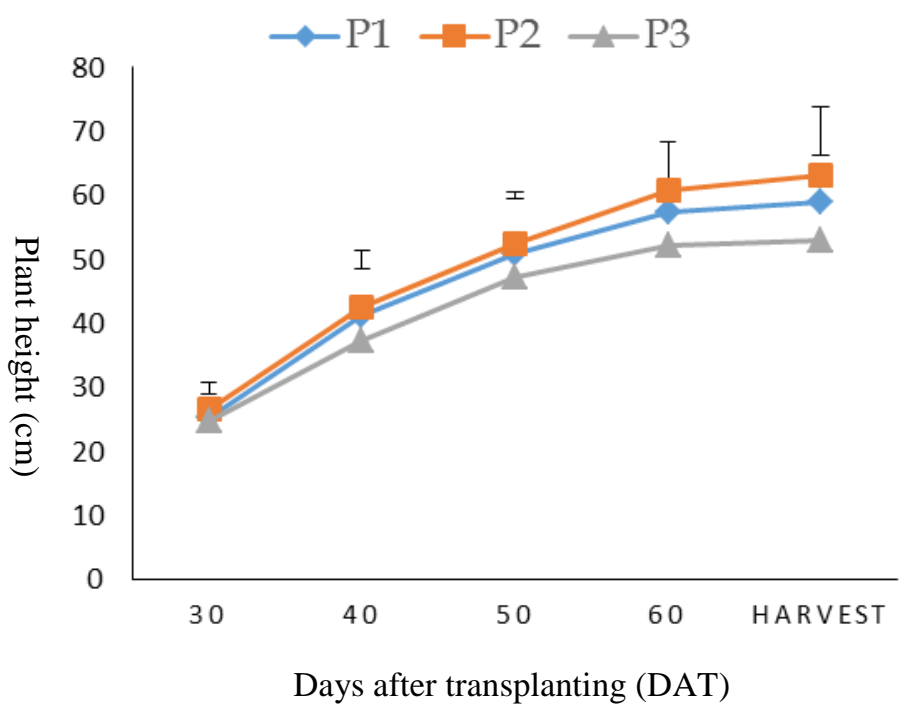

Fig. 1. Effect of planting date on plant height at different days after transplanting of cauliflower. The vertical bars represent LSD at $1 \%$ level of probability

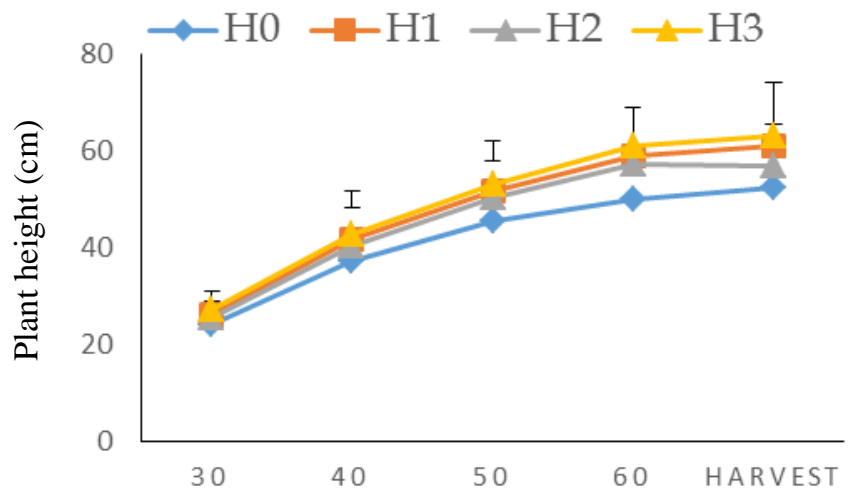

Davs after transwlanting (DAT)

Fig. 2. Effect of hormone on plant height at different days after transplanting of cauliflower. The vertical bars represent LSD at $1 \%$ level of probability.

It was found that the tallest plant 27.27, 45.58, 54.96, 65.78 and $65.96 \mathrm{~cm}$ was found from the treatment combination of $\mathrm{P}_{2} \mathrm{H}_{3}$ (planting at 15 November $\times 10$ ppm IAA $\left.+70 \mathrm{ppm} \mathrm{GA}_{3}\right)$ respectively whereas the shortest plant 22.55, 31.53, 41.80, 42.75 and $45.44 \mathrm{~cm}$ was recorded from $\mathrm{P}_{3} \mathrm{H}_{0}$ (planting at 1 December $\times$ no hormone) at 30, 40, 50, 60 DAT and at harvest, respectively (Table 1 ). 
Table 1. Combined effect of planting date and growth hormones on plant height of cauliflower at different days after transplanting

\begin{tabular}{|c|c|c|c|c|c|}
\hline \multirow{2}{*}{ Treatment } & \multicolumn{5}{|c|}{ Plant height (cm) at different DAT } \\
\cline { 2 - 6 } & $\mathbf{3 0}$ & $\mathbf{4 0}$ & $\mathbf{5 0}$ & $\mathbf{6 0}$ & Harvest \\
\hline $\mathrm{P}_{1} \mathrm{H}_{0}$ & 23.61 & 38.81 & 46.95 & 51.9 & 52.71 \\
\hline $\mathrm{P}_{1} \mathrm{H}_{1}$ & 24.1 & 41.58 & 49.61 & 58.2 & 57.69 \\
\hline $\mathrm{P}_{1} \mathrm{H}_{2}$ & 27.04 & 40.45 & 53.36 & 58.93 & 60.51 \\
\hline $\mathrm{P}_{1} \mathrm{H}_{3}$ & 26.81 & 43.77 & 53.45 & 61.17 & 64.6 \\
\hline $\mathrm{P}_{2} \mathrm{H}_{0}$ & 24.09 & 41.34 & 47.73 & 55.57 & 58.99 \\
\hline $\mathrm{P}_{2} \mathrm{H}_{1}$ & 24.76 & 43.16 & 52.78 & 63.85 & 62.69 \\
\hline $\mathrm{P}_{2} \mathrm{H}_{2}$ & 25.79 & 40.57 & 50.74 & 57.77 & 60.38 \\
\hline $\mathrm{P}_{2} \mathrm{H}_{3}$ & 27.27 & 45.58 & 54.96 & 65.78 & 65.96 \\
\hline $\mathrm{P}_{3} \mathrm{H}_{0}$ & 22.55 & 31.53 & 41.80 & 42.75 & 45.44 \\
\hline $\mathrm{P}_{3} \mathrm{H}_{1}$ & 24.88 & 40.32 & 49.06 & 55.03 & 58.32 \\
\hline $\mathrm{P}_{3} \mathrm{H}_{2}$ & 23.43 & 39.83 & 47.37 & 55.38 & 50.31 \\
\hline $\mathrm{P}_{3} \mathrm{H}_{3}$ & 26.9 & 38.68 & 50.96 & 55.89 & 58.42 \\
\hline LSD at 5\% & 0.94 & 1.46 & 1.75 & 3.50 & 3.74 \\
\hline LSD at 1\% & 1.27 & 1.96 & 2.37 & 4.76 & 5.08 \\
\hline Significance level & $* *$ & $* *$ & $* *$ & $* *$ & $* *$ \\
\hline
\end{tabular}

$* *=$ Significant at $1 \%$ level of probability $\quad *=$ Significant at $5 \%$ level of probability

$\mathrm{P}_{1}$ : Planting on 1 November

$\mathrm{H}_{0}$ : No Hormone

$\mathrm{P}_{2}$ : Planting on 15 November

$\mathrm{H}_{1}: 10 \mathrm{ppm}$ IAA

$\mathrm{P}_{3}$ : Planting on 1 December

$\mathrm{H}_{2}: \quad 70 \mathrm{ppm} \mathrm{GA}_{3}$

$\mathrm{H}_{3}: 10 \mathrm{ppm} \mathrm{IAA}+70$ ppm GA

\section{Number of leaves per plant}

At 30, 40, 50,60 DAT and at harvest the maximum number of leaves per plant 12.43, 15.91, 18.74, 23.40 and 24.13 was observed in $\mathrm{P}_{2}$ (planting at 15 November) which was closely followed $11.95,14.96$, $18.06,22.23$ and 22.07 by $\mathrm{P}_{1}$ (planting at 1 November). Again, at the same DAT the minimum number of leaves per plant 11.21, 14.25, 16.27, 19.20 and 20.23 was found from $\mathrm{P}_{3}$ (planting at 1 December), respectively (Fig. 3). At 30, 40, 50, 60 DAT and at harvest the maximum number of leaves per plant 13.54, 15.68, 18.66, 23.06 and 23.66 was recorded from $\mathrm{H}_{3}$ (10 ppm IAA $+70 \mathrm{ppm} \mathrm{GA} 3$ ) which was followed 12.81, 15.35, $18.11,22.31$ and 22.84 by $\mathrm{H}_{1}$ (10 ppm IAA). Again, the minimum number of leaves per plant $(9.9,14.25,16.38$, 19.41 and 19.82) was found from $\mathrm{H}_{0}$ (no hormone) for the same DAT, respectively (Fig. 4).
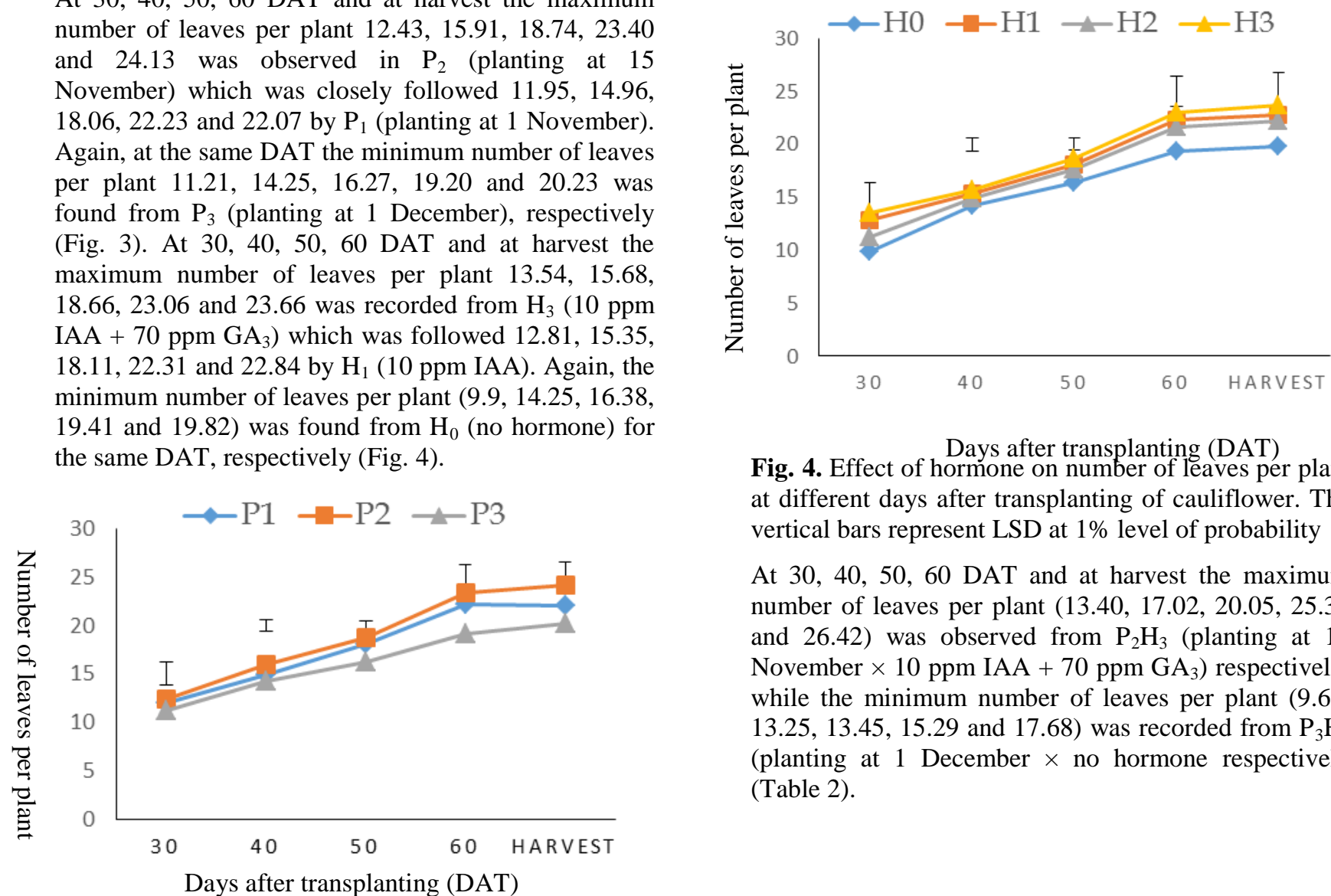

Days after transplanting (DAT)

Fig. 4. Effect of hormone on number of leaves per plant at different days after transplanting of cauliflower. The vertical bars represent LSD at $1 \%$ level of probability

At 30, 40, 50, 60 DAT and at harvest the maximum number of leaves per plant $(13.40,17.02,20.05,25.38$ and 26.42) was observed from $\mathrm{P}_{2} \mathrm{H}_{3}$ (planting at 15 November $\times 10 \mathrm{ppm}$ IAA $+70 \mathrm{ppm} \mathrm{GA} 3$ ) respectively, while the minimum number of leaves per plant $(9.60$, $13.25,13.45,15.29$ and 17.68) was recorded from $\mathrm{P}_{3} \mathrm{H}_{0}$ (planting at 1 December $\times$ no hormone respectively (Table 2).

Fig. 3. Effect of planting date on number of leaves per plant at different days after transplanting of cauliflower. The vertical bars represent LSD at $1 \%$ level of probability 
Table 2. Combined effect of planting date and growth hormones on number of leaves per plant of cauliflower at different days after transplanting

\begin{tabular}{|c|c|c|c|c|c|}
\hline \multirow{2}{*}{ Treatment } & \multicolumn{5}{|c|}{ Number of leaves per plant at different DAT } \\
\cline { 2 - 6 } & $\mathbf{3 0}$ & $\mathbf{4 0}$ & $\mathbf{5 0}$ & $\mathbf{6 0}$ & Harvest \\
\hline $\mathrm{P}_{1} \mathrm{H}_{0}$ & 9.70 & 13.95 & 17.62 & 20.85 & 19.45 \\
\hline $\mathrm{P}_{1} \mathrm{H}_{1}$ & 11.00 & 15.65 & 18.45 & 22.72 & 22.55 \\
\hline $\mathrm{P}_{1} \mathrm{H}_{2}$ & 11.43 & 14.98 & 16.82 & 21.19 & 22.72 \\
\hline $\mathrm{P}_{1} \mathrm{H}_{3}$ & 12.70 & 15.28 & 19.35 & 24.18 & 23.58 \\
\hline $\mathrm{P}_{2} \mathrm{H}_{0}$ & 10.40 & 15.55 & 18.08 & 22.08 & 22.32 \\
\hline $\mathrm{P}_{2} \mathrm{H}_{1}$ & 12.63 & 16.12 & 18.09 & 23.09 & 25.35 \\
\hline $\mathrm{P}_{2} \mathrm{H}_{2}$ & 11.10 & 14.92 & 18.75 & 23.08 & 22.45 \\
\hline $\mathrm{P}_{2} \mathrm{H}_{3}$ & 13.40 & 17.02 & 20.05 & 25.38 & 26.42 \\
\hline $\mathrm{P}_{3} \mathrm{H}_{0}$ & 9.60 & 13.25 & 13.45 & 15.29 & 17.68 \\
\hline $\mathrm{P}_{3} \mathrm{H}_{1}$ & 11.80 & 14.28 & 17.78 & 21.12 & 20.62 \\
\hline $\mathrm{P}_{3} \mathrm{H}_{2}$ & 10.10 & 14.72 & 17.28 & 20.78 & 21.65 \\
\hline $\mathrm{P}_{3} \mathrm{H}_{3}$ & 13.23 & 14.75 & 16.59 & 19.62 & 20.98 \\
\hline LSD at 5\% & 1.16 & 0.53 & 0.48 & 1.21 & 1.51 \\
\hline LSD at 1\% & 1.58 & 0.73 & 0.65 & 1.65 & 2.05 \\
\hline Significance level & $*$ & $*$ & $* *$ & & $*$ \\
\hline
\end{tabular}

$* *=$ Significant at $1 \%$ level of probability $\quad *=$ Significant at $5 \%$ level of probability

$\mathrm{P}_{1}$ : Planting on 1 November

$\mathrm{P}_{2}$ : Planting on 15 November

$\mathrm{H}_{0}$ : No Hormone

$\mathrm{P}_{3}$ : Planting on 1 December

$\mathrm{H}_{1}$ : 10 ppm IAA

$\mathrm{H}_{2}: \quad 70 \mathrm{ppm} \mathrm{GA}_{3}$

$\mathrm{H}_{3}: 10$ ppm IAA +70 ppm GA

Table 3. Effect of planting date and growth hormones on yield contributing characters of cauliflower

\begin{tabular}{|c|c|c|c|c|c|}
\hline Treatment & $\begin{array}{l}\text { Days from } \\
\text { transplanting to } \\
\text { curd initiation }\end{array}$ & $\begin{array}{l}\text { Days from } \\
\text { transplanting to } \\
50 \% \text { curd } \\
\text { formation }\end{array}$ & $\begin{array}{c}\text { Days from } \\
\text { transplanting to } \\
\text { harvest }\end{array}$ & $\begin{array}{l}\text { Stem length } \\
(\mathrm{cm})\end{array}$ & $\begin{array}{c}\text { Stem } \\
\text { Diameter }(\mathrm{cm})\end{array}$ \\
\hline \multicolumn{6}{|c|}{ Planting date } \\
\hline $\mathrm{P}_{1}$ & 47.06 & 51.71 & 66.45 & 9.80 & 1.40 \\
\hline $\mathrm{P}_{2}$ & 40.07 & 45.49 & 61.18 & 11.49 & 1.76 \\
\hline $\mathrm{P}_{3}$ & 42.28 & 47.62 & 63.75 & 9.03 & 1.09 \\
\hline LSD at $5 \%$ & 1.34 & 1.43 & 1.61 & 0.37 & 0.02 \\
\hline LSD at $1 \%$ & 1.82 & 1.94 & 2.19 & 0.51 & 0.03 \\
\hline Significant Level & $* *$ & $* *$ & $* *$ & $* *$ & $* *$ \\
\hline \multicolumn{6}{|c|}{ Growth hormone } \\
\hline $\mathrm{H}_{0}$ & 45.97 & 50.24 & 67.41 & 9.43 & 1.35 \\
\hline $\mathrm{H}_{1}$ & 44.34 & 48.36 & 65.29 & 10.32 & 1.53 \\
\hline $\mathrm{H}_{2}$ & 41.67 & 48.14 & 62.74 & 9.53 & 1.34 \\
\hline $\mathrm{H}_{3}$ & 40.56 & 46.36 & 59.74 & 11.16 & 1.46 \\
\hline LSD at $5 \%$ & 1.55 & 1.65 & 1.86 & 0.43 & 0.03 \\
\hline LSD at $1 \%$ & 2.11 & 2.25 & 2.53 & 0.59 & 0.04 \\
\hline Significant Level & $* *$ & $* *$ & $* *$ & $* *$ & $* *$ \\
\hline \multicolumn{6}{|c|}{$* *=$ Significant at $1 \%$ level of probability $\quad *=$ Significant at $5 \%$ level of probability } \\
\hline \multicolumn{2}{|c|}{$\begin{array}{ll}\mathrm{P}_{1} \text { : Planting on } 1 \text { November } & \mathrm{H}_{0} \\
\mathrm{P}_{2} \text { : Planting on } 15 \text { November } & \mathrm{H}_{1} \\
\mathrm{P}_{3} \text { : Planting on } 1 \text { December } & \mathrm{H}_{2}\end{array}$} & $\begin{array}{l}\text { No Hormone } \\
10 \text { ppm IAA } \\
70 \text { ppm GA } \text { GA }_{3} \\
10 \text { ppm IAA + } 70 \text { p }\end{array}$ & $\mathrm{n} \mathrm{GA}_{3}$ & & \\
\hline
\end{tabular}

\section{Curd weight with leaf}

The highest curd weight with leaf $2.05 \mathrm{~kg}$ was obtained from $\mathrm{P}_{2}$ (planting on 15 November) and the lowest curd weight with leaf $1.34 \mathrm{~kg}$ was recorded from $\mathrm{P}_{3}$ (planting on 1 December) (Table 3 ).

The highest curd weight with leaf $1.88 \mathrm{~kg}$ was observed from $\mathrm{H}_{3}\left(10\right.$ ppm IAA +70 ppm $\left.\mathrm{GA}_{3}\right)$ which was closely followed $1.82 \mathrm{~kg}$ by $\mathrm{H}_{1}(10 \mathrm{ppm}$ IAA). The lowest curd weight with leaf $1.69 \mathrm{~kg}$ was found from $\mathrm{H}_{0}$ (no hormone) (Table 3).

The highest curd weight with leaf $2.52 \mathrm{~kg}$ was obtained from the treatment combination of $\mathrm{P}_{2} \mathrm{H}_{3}$ (planting on 15 November $\times 10$ ppm IAA +70 ppm $\mathrm{GA}_{3}$ ) where as the lowest curd weight with leaf $1.01 \mathrm{~kg}$ was observed from $\mathrm{P}_{3} \mathrm{H}_{0}$ (planting 1 December x no hormone) (Table 4). 
Table 4. Combined effect of planting date and growth hormones on yield contributing characters of cauliflower

\begin{tabular}{|c|c|c|c|c|c|}
\hline Treatment & $\begin{array}{c}\text { Days from } \\
\text { transplanting to } \\
\text { curd initiation }\end{array}$ & $\begin{array}{c}\text { Days from } \\
\text { transplanting } \\
\text { to 50\% curd } \\
\text { formation }\end{array}$ & $\begin{array}{c}\text { Days from } \\
\text { transplanting } \\
\text { to harvest }\end{array}$ & $\begin{array}{c}\text { Stem length } \\
\text { (cm) }\end{array}$ & $\begin{array}{c}\text { Stem } \\
\text { Diameter } \\
\text { (cm) }\end{array}$ \\
\hline $\mathrm{P}_{1} \mathrm{H}_{0}$ & 50.56 & 54.78 & 69.8 & 8.57 & 1.59 \\
\hline $\mathrm{P}_{1} \mathrm{H}_{1}$ & 46.95 & 53.22 & 63.83 & 9.98 & 1.38 \\
\hline $\mathrm{P}_{1} \mathrm{H}_{2}$ & 45.79 & 51.08 & 68.6 & 9.82 & 1.48 \\
\hline $\mathrm{P}_{1} \mathrm{H}_{3}$ & 44.95 & 50.78 & 63.6 & 10.85 & 1.16 \\
\hline $\mathrm{P}_{2} \mathrm{H}_{0}$ & 43.62 & 47.88 & 65.83 & 11.14 & 1.72 \\
\hline $\mathrm{P}_{2} \mathrm{H}_{1}$ & 41.45 & 43.42 & 66.46 & 12.31 & 1.46 \\
\hline $\mathrm{P}_{2} \mathrm{H}_{2}$ & 36.28 & 45.12 & 55.93 & 9.19 & 1.9 \\
\hline $\mathrm{P}_{2} \mathrm{H}_{3}$ & 35.95 & 45.55 & 56.5 & 13.35 & 1.96 \\
\hline $\mathrm{P}_{3} \mathrm{H}_{0}$ & 43.79 & 51.08 & 67.8 & 8.59 & 0.74 \\
\hline $\mathrm{P}_{3} \mathrm{H}_{1}$ & 44.62 & 48.45 & 65.6 & 8.67 & 1.75 \\
\hline $\mathrm{P}_{3} \mathrm{H}_{2}$ & 42.95 & 47.22 & 62.5 & 9.58 & 0.84 \\
\hline $\mathrm{P}_{3} \mathrm{H}_{3}$ & 37.79 & 42.75 & 59.13 & 9.3 & 1.26 \\
\hline LSD at 5\% & 2.69 & 2.86 & 3.22 & 0.75 & 0.05 \\
\hline LSD at $1 \%$ & 3.65 & 3.89 & 4.38 & 1.02 & 0.07 \\
\hline Significance level & $* *$ & $* *$ & $* *$ & $* *$ & $* *$ \\
\hline
\end{tabular}

$* *=$ Significant at $1 \%$ level of probability

$\mathrm{P}_{1}$ : Planting on 1 November

$\mathrm{P}_{2}$ : Planting on 15 November

$\mathrm{P}_{3}$ : Planting on 1 December

\author{
$\mathrm{H}_{0}$ : No Hormone \\ $\mathrm{H}_{1}$ : 10 ppm IAA \\ $\mathrm{H}_{2}: 70$ ppm GA \\ $\mathrm{H}_{3}: 10$ ppm IAA +70 ppm GA 3
}

Table 5. Effect of planting date and growth hormones on yield contributing characters and yield of cauliflower

\begin{tabular}{|c|c|c|c|c|c|}
\hline Treatment & $\begin{array}{l}\text { Curd weight } \\
\text { with leaf } \\
(\mathrm{kg})\end{array}$ & $\begin{array}{c}\text { Pure curd } \\
\text { height } \\
(\mathrm{cm})\end{array}$ & $\begin{array}{c}\text { Curd Diameter } \\
(\mathrm{cm})\end{array}$ & $\begin{array}{c}\text { Marketable } \\
\text { yield } \\
\left(\text { kg plant }^{-1}\right)\end{array}$ & $\begin{array}{c}\text { Marketable } \\
\text { yield } \\
\left(\mathrm{t} \mathrm{ha}^{-1}\right)\end{array}$ \\
\hline \multicolumn{6}{|c|}{ Planting date } \\
\hline $\mathrm{P}_{1}$ & 1.84 & 13.83 & 21.68 & 1.08 & 27.05 \\
\hline $\mathrm{P}_{2}$ & 2.05 & 14.53 & 22.25 & 1.12 & 28.11 \\
\hline $\mathrm{P}_{3}$ & 1.34 & 11.93 & 17.66 & 1.04 & 25.98 \\
\hline LSD at $5 \%$ & 0.06 & 0.66 & 0.96 & 0.02 & 0.56 \\
\hline LSD at $1 \%$ & 0.08 & 0.89 & 1.30 & 0.03 & 0.77 \\
\hline Significant Level & $* *$ & $* *$ & $* *$ & $* *$ & $* *$ \\
\hline \multicolumn{6}{|c|}{ Growth hormone } \\
\hline $\mathrm{H}_{0}$ & 1.69 & 12.16 & 18.60 & 0.94 & 23.47 \\
\hline $\mathrm{H}_{1}$ & 1.82 & 14.07 & 21.35 & 1.12 & 26.95 \\
\hline $\mathrm{H}_{2}$ & 1.84 & 12.77 & 19.80 & 1.08 & 27.89 \\
\hline $\mathrm{H}_{3}$ & 1.88 & 14.71 & 22.39 & 1.20 & 29.88 \\
\hline LSD at $5 \%$ & 0.06 & 0.76 & 1.11 & 0.03 & 0.65 \\
\hline LSD at $1 \%$ & 0.09 & 1.03 & 1.50 & 0.04 & 0.89 \\
\hline Significant Level & $* *$ & $* *$ & $* *$ & $* *$ & $* *$ \\
\hline \multicolumn{6}{|c|}{$* *=$ Significant at $1 \%$ level of probability $\quad *=$ Significant at $5 \%$ level of probability } \\
\hline \multirow{3}{*}{\multicolumn{2}{|c|}{$\begin{array}{l}\mathrm{P}_{1} \text { : Planting on } 1 \text { November } \\
\mathrm{P}_{2} \text { : Planting on } 15 \text { November } \\
\mathrm{P}_{3} \text { : Planting on } 1 \text { December }\end{array}$}} & \multicolumn{4}{|c|}{ No Hormone } \\
\hline & & \multicolumn{4}{|l|}{10 ppm IAA } \\
\hline & & \multicolumn{4}{|l|}{$70 \mathrm{ppm} \mathrm{GA} 3$} \\
\hline
\end{tabular}

\section{Pure curd height}

The maximum pure curd height $14.53 \mathrm{~cm}$ was recorded from $\mathrm{P}_{2}$ (planting 15 November) and the minimum pure curd height $11.93 \mathrm{~cm}$ was found from $\mathrm{P}_{3}$ (planting on 1 December) (Table 5). The maximum pure curd height $14.71 \mathrm{~cm}$ was recorded from $\mathrm{H}_{3}(10 \mathrm{ppm} \mathrm{IAA}+70 \mathrm{ppm}$ $\mathrm{GA}_{3}$ ) which was statistically identical $14.07 \mathrm{~cm}$ with $\mathrm{H}_{1}$ (10 ppm IAA). The minimum pure curd height 12.16 $\mathrm{cm}$ was recorded from $\mathrm{H}_{0}$ (no hormone) which was statistically similar $12.77 \mathrm{~cm}$ to $\mathrm{H}_{2}\left(70 \mathrm{ppm} \mathrm{GA}_{3}\right)$ (Table 5).

The maximum pure curd height $14.28 \mathrm{~cm}$ was found from the treatment combination of $\mathrm{P}_{2} \mathrm{H}_{3}$ (planting on 15 November x 10 ppm IAA + 70 ppm $\left.\mathrm{GA}_{3}\right)$ where as the minimum pure curd height $(9.99 \mathrm{~cm})$ was found from $\mathrm{P}_{1} \mathrm{H}_{0}$ (planting on 1 November $\mathrm{x}$ no hormone) (Table $6)$. 


\section{Curd diameter}

The maximum curd diameter $22.25 \mathrm{~cm}$ was found from $\mathrm{P}_{2}$ (planting at 15 November) which was statistically similar $21.68 \mathrm{~cm}$ to $\mathrm{P}_{1}$ (planting on 1 November) and the minimum curd diameter $17.66 \mathrm{~cm}$ was obtained from $\mathrm{P}_{3}$ (planting on 1 December) (Table 5).

Table 6. Combined effect of planting date and growth hormones on yield contributing characters and yield of cauliflower

\begin{tabular}{|c|c|c|c|c|c|}
\hline Treatment & $\begin{array}{c}\text { Curd weight } \\
\text { with leaf } \\
(\mathrm{kg}) \\
\end{array}$ & $\begin{array}{l}\text { Pure curd } \\
\text { height } \\
(\mathrm{cm})\end{array}$ & $\begin{array}{l}\text { Curd Diameter } \\
(\mathbf{c m})\end{array}$ & $\begin{array}{c}\text { Marketable } \\
\text { yield } \\
\left(\text { kg plant }^{-1}\right) \\
\end{array}$ & $\begin{array}{c}\text { Marketable } \\
\text { yield } \\
\left(\text { t ha }^{-1}\right) \\
\end{array}$ \\
\hline $\mathrm{P}_{1} \mathrm{H}_{0}$ & 1.92 & 9.99 & 18.27 & 0.98 & 24.58 \\
\hline $\mathrm{P}_{1} \mathrm{H}_{1}$ & 1.62 & 11.86 & 19.85 & 1.05 & 26.17 \\
\hline $\mathrm{P}_{1} \mathrm{H}_{2}$ & 2.13 & 12.31 & 23.98 & 1.10 & 27.60 \\
\hline $\mathrm{P}_{1} \mathrm{H}_{3}$ & 1.70 & 13.58 & 24.65 & 1.19 & 29.87 \\
\hline $\mathrm{P}_{2} \mathrm{H}_{0}$ & 2.16 & 12.74 & 20.05 & 0.96 & 24.08 \\
\hline $\mathrm{P}_{2} \mathrm{H}_{1}$ & 2.02 & 11.77 & 24.32 & 1.22 & 30.40 \\
\hline $\mathrm{P}_{2} \mathrm{H}_{2}$ & 2.31 & 13.33 & 19.41 & 1.08 & 26.95 \\
\hline $\mathrm{P}_{2} \mathrm{H}_{3}$ & 2.52 & 14.28 & 25.75 & 1.24 & 31.03 \\
\hline $\mathrm{P}_{3} \mathrm{H}_{0}$ & 1.01 & 13.77 & 16.02 & 0.87 & 21.75 \\
\hline $\mathrm{P}_{3} \mathrm{H}_{1}$ & 2.01 & 13.60 & 18.9 & 1.08 & 27.12 \\
\hline $\mathrm{P}_{3} \mathrm{H}_{2}$ & 1.09 & 11.69 & 16.48 & 1.05 & 26.31 \\
\hline $\mathrm{P}_{3} \mathrm{H}_{3}$ & 1.25 & 10.29 & 18.27 & 1.15 & 28.74 \\
\hline LSD at $5 \%$ & 0.12 & 1.31 & 1.92 & 0.05 & 1.13 \\
\hline LSD at $1 \%$ & 0.16 & 1.79 & 2.61 & 0.07 & 1.54 \\
\hline Significance level & $* *$ & $*$ & $* *$ & $* *$ & $* *$ \\
\hline \multicolumn{6}{|c|}{$* *=$ Significant at $1 \%$ level of probability } \\
\hline \multirow{3}{*}{\multicolumn{2}{|c|}{$\begin{array}{l}\mathrm{P}_{1} \text { : Planting on } 1 \text { November } \\
\mathrm{P}_{2} \text { : Planting on } 15 \text { November } \\
\mathrm{P}_{3} \text { : Planting on } 1 \text { December }\end{array}$}} & \multicolumn{4}{|l|}{ No Hormone } \\
\hline & & \multicolumn{4}{|l|}{10 ppm IAA } \\
\hline & & \multicolumn{4}{|l|}{70 ppm GA 3} \\
\hline
\end{tabular}

The maximum curd diameter $22.39 \mathrm{~cm}$ was recorded from $\mathrm{H}_{3}\left(10 \mathrm{ppm}\right.$ IAA $\left.+70 \mathrm{ppm} \mathrm{GA}_{3}\right)$ which was statistically identical $21.35 \mathrm{~cm}$ with $\mathrm{H}_{1}$ (10 ppm IAA), whereas, the minimum curd diameter $18.60 \mathrm{~cm}$ was recorded from $\mathrm{H}_{0}$ (no hormone) which was closely followed $19.80 \mathrm{~cm}$ by $\mathrm{H}_{2}(70 \mathrm{ppm} \mathrm{GA}$ ) (Table 5). The maximum curd diameter $25.75 \mathrm{~cm}$ was found from $\mathrm{P}_{2} \mathrm{H}_{3}$ (planting on 15 November x 10 ppm IAA $+70 \mathrm{ppm}$ $\mathrm{GA}_{3}$ ) where as the minimum curd diameter $16.02 \mathrm{~cm}$ was recorded from the treatment combination of $\mathrm{P}_{3} \mathrm{H}_{0}$ (planting on 1 December $\mathrm{x}$ no hormone) that is shown in (Table 6).

\section{Marketable yield per plant}

The highest marketable yield per plant $1.12 \mathrm{~kg}$ was recorded from $\mathrm{P}_{2}$ (planting on 15 November) which was closely followed $1.08 \mathrm{~kg}$ by $\mathrm{P}_{1}$ (planting 1 November) and the lowest marketable yield per plant $1.04 \mathrm{~kg}$ was obtained from $\mathrm{P}_{3}$ (planting on 1 December) that is shown in (Table 5).

The highest marketable yield per plan. $1.20 \mathrm{~kg}$ was recorded from $\mathrm{H}_{3}\left(10\right.$ ppm IAA +70 ppm $\left.\mathrm{GA}_{3}\right)$ which was closely followed $1.12 \mathrm{~kg}$ by $\mathrm{H}_{1}$ (10ppm IAA) and the lowest marketable yield per plant $0.94 \mathrm{~kg}$ was found from and $\mathrm{H}_{0}$ (no hormone) (Table 5).

The highest marketable yield per plant $1.24 \mathrm{~kg}$ was recorded from the treatment combination of $\mathrm{P}_{2} \mathrm{H}_{3}$ (planting on 15 December x 10 ppm IAA +70 ppm $\mathrm{GA}_{3}$ ) which was statistical similar to $\mathrm{P}_{2} \mathrm{H}_{1} 1.22 \mathrm{~kg}$ and $\mathrm{P}_{1} \mathrm{H}_{3} 1.19 \mathrm{~kg}$ and the lowest marketable yield per plant $0.87 \mathrm{~kg}$ was recorded from $\mathrm{P}_{3} \mathrm{H}_{0}$ (planting on 1 December $\mathrm{x}$ no hormone) (Table 8).

\section{Marketable yield per hectare}

The highest marketable yield $28.11 \mathrm{t} \mathrm{ha}^{-1}$ was observed from $\mathrm{P}_{2}$ (planting on 15 November) and the lowest marketable yield $25.98 \mathrm{t} \mathrm{ha}^{-1}$ was found from $\mathrm{P}_{3}$ (planting on 1 December) (Table 5) and (Fig. 5).

The highest marketable yield $29.88 \mathrm{t} / \mathrm{ha}$ was obtained from $\mathrm{H}_{3}\left(10\right.$ ppm IAA +70 ppm $\left.\mathrm{GA}_{3}\right)$ which was closely followed $\left(27.89 \mathrm{t} \mathrm{ha}^{-1}\right)$ by $\mathrm{H}_{2}\left(70 \mathrm{ppm} \mathrm{GA}_{3}\right)$ (Table 5) and (Fig. 6).

The highest marketable yield $31.03 \mathrm{t} \mathrm{ha}^{-1}$ was observed from $\mathrm{P}_{2} \mathrm{H}_{3}$ (planting 15 November x 10 ppm IAA + 70 ppm $\mathrm{GA}_{3}$ ), while the lowest marketable yield $21.75 \mathrm{tha}$ ${ }^{1}$ was found from $\mathrm{P}_{3} \mathrm{H}_{0}$ (planting on 1 December $\mathrm{x}$ no hormone) (Table 6) and (Fig. 7).

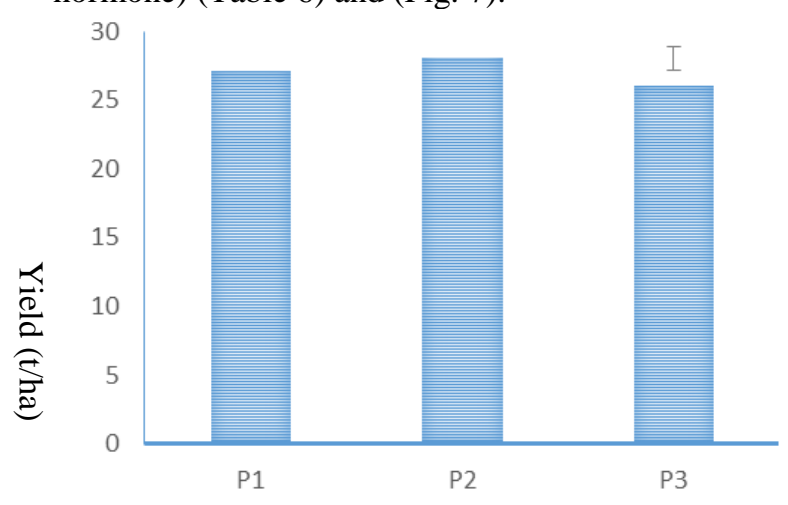

Planting date

Fig. 5. Effect of planting date on yield per hectare of cauliflower. The vertical bar represents LSD at $1 \%$ level of probability 
$\mathrm{P}_{1}$ : Planting on 1 November; $\mathrm{P}_{2}$ : Planting on 15

November; $\mathrm{P}_{3}$ : Planting on 1 December

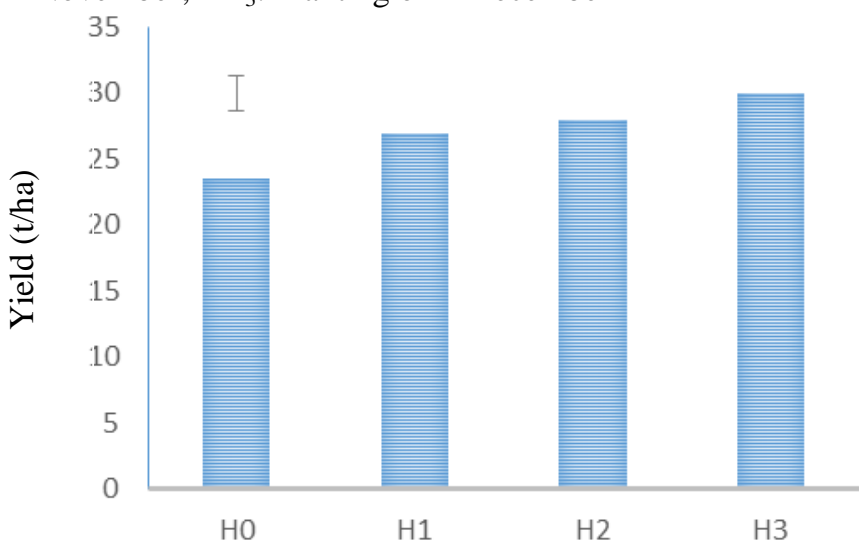

Plant growth hormones

Fig. 6. Etfect of growth hormone on yield per hectare of cauliflower. The vertical bar represents LSD at $1 \%$ level of probability

$\mathrm{H}_{0}$ : No Hormone $\mathrm{H}_{1}: 10$ ppm IAA

$\mathrm{H}_{2}: 70$ ppm GA $\mathrm{H}_{3}: 10$ ppm IAA +70 ppm GA

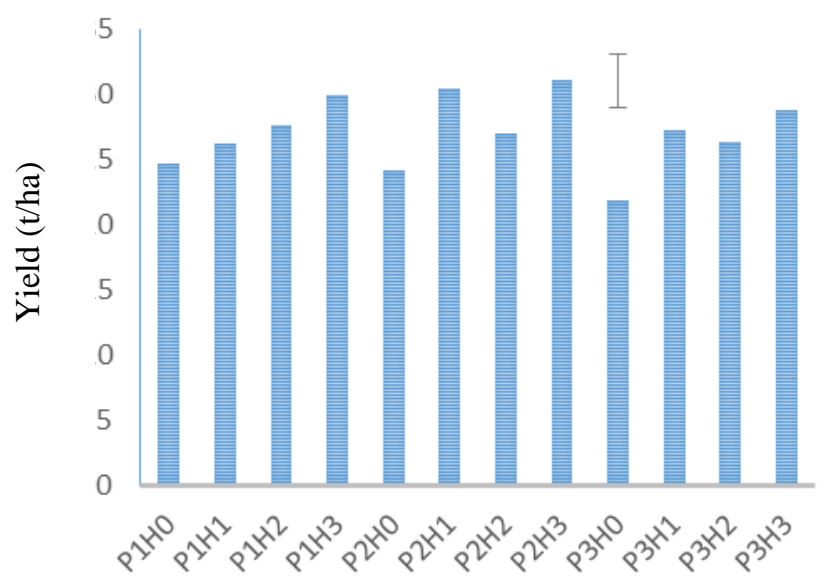

Planting date

Fig. 7. Combined effect of planting date and growth hormones on yield per hectare of cauliflower. The vertical bar represents LSD at $1 \%$ level of probability.

$\mathrm{P}_{1}$ : Planting on 1 November $\mathrm{H}_{0}$ : No Hormone

$\mathrm{P}_{2}$ : Planting on 15 November $\mathrm{H}_{1}: 10$ ppm IAA

$\mathrm{P}_{3}$ : Planting on 1 December $\mathrm{H}_{2}: 70 \mathrm{ppm} \mathrm{GA}_{3}$ $\mathrm{H}_{3}: 10$ ppm IAA + 70 ppm GA

\section{Conclusions}

From the present experiment it is clear that growth and yield of Cauliflower largely depend upon the planting date and growth hormone. These two factors either singly or in combination influence the growth, quality and yield of the crop. In this experiment, the highest yield per hectare was obtained from the combination with planting on 15 November $x 10$ ppm IAA + 70 ppm $\mathrm{GA}_{3}$. On the other hand, the lowest yield per hectare was obtained from the combination with planting on 15 December x No hormone.

\section{References}

Abdalla, I. M.; Helal, R. M. and Zaki, M. E. 1980. Studies on the effect of some growth hormones on yield and quality of cauliflower. Annual Agricultural Science, 12: 199-208.

Aditya, N. and Fordham, S. E. 1995. Effects of cold exposure and $\mathrm{GA}_{3}$ during early growth stages on the date of flowering of the tropical cauliflower. Indian Journal of Plant Physiology, 32(1): 111-115.

Ara, N.; Kaisar, M. O.; Khalequzzaman, K. M.; Kohmoor, H. and Ahamed, 2009. Effect of different dates of planting and lines on the growth, yield and yield contributing characteristics of cauliflower. Journal of Soil Nature, 3(1): 16-19.

Badawi, M. A. and Sahhar, K. F. E. 1979. Influence of some growth substances on different characters of cabbage. Egypt Journal of Horticulture, 6(2): 221235.

Biswas, A. K. and Mandal, S. K. 1994. Manipulation of senescence, source-sink relationship and yield of growth regulating chemicals. Indian Journal of Plant Physiology, 31(2): 152-157.

Chauhan, K. S. and Bordia, N. S. 1971. Effect of gibberellic acid, beta- napthoacetic acid and 2,4dichlorophenoxyacetic acid as pre sowing seed treatment on growth and yield of cabbage (Brassica oleracea var capitata L.). Indian Journal of Horticulture, pp. 57-63.

Chhonkar, V. S. and Jha, R. N. 1963. The use of starters and plant growth hormones in transplanting of cabbage and their response on growth and yield. Indian Journal of Horticulture, 20(2): 122-128.

Csizinszky, A. A. 1996. Optimum planting time, plant spacing, and nitrogen and potassium rates to maximize yield of cauliflower. Horticulture Science, 31(6): 930-933.

Denisova, A. Z. and Luponovich, I. S. 1962. The effects of gibberellic acid on the mineral nutrition of plants. Soil Science Institute Bangladesh Soil Science Research Agriculture Minsk USSR, 8(4): 360-364.

Dharmender, K.; Hujar, K. D.; Paliwal, R. and Kumar, D. 1996. Yield and yield attributes of cabbage as influenced by $\mathrm{GA}_{3}$ and NAA. Crop Research Hisar, 12(1): 120-122.

Dutta, J. P. 1999. Effect of dates of planting on the performance of different groups of cauliflower under the condition of North Bank Plains Zone of Assam. Journal of the Agricultural Science Society of North East India, 12(1): 38-42.

FAO. 1988. Production Year Book. Food and Agricultural of the United Nations, Rome, Italy. 42: 190-193.

FAO. 2007. Production year book. Food and Agricultural Organization of the United Nations, Rome, Italy. p. 141.

Gautam, B. P.; Shadeque, A. and Chakrabarty, B. K. 2001. Effect of sowing date, variety and method of seed production on the growth and seed yield of early cauliflower. Horticultural Journal, 14(2): 171179. 
Gautam, B. P.; Shadeque, A. and Saikia, L. 1998. Effect of sowing dates and varieties on growth and yield of early cauliflower. Vegetable Science, 25(1): 1-4.

Ghanti, P. and Mallik, S. C. 1995. Influence of planting time on stem growth and curd compactness in early cauliflower. Orissa Journal of Horticulture, 23(1): $37-44$.

Gomez, K. A. and Gomez, A. A 1984. Statistical Procedure for Agricultural Research (2nd edition). Rice Research Institute. A Willey Institute of Science, pp. 28-192.

Guo, D. P.; Shah, G. A.; Zeng, G. W. and Zheng, S. 2004. The interaction of plant growth regulators and vernalization on the growth and flowering of cauliflower (Brassica oleracea var. botrytis). Plant Growth Regulation, 43(2): 163-171.

Islam, M. A.; Diddiqua, A. and Kashem, M. A. 1993. Effect of growth regulators on growth, yield and ascorbic acid content of cabbage. Bangladesh Journal of Agricultural Science, 20(1): 21-27.

Jana, J. C. and Mukhopadhyay, T. P. 2002. Effect of sowing dates and varieties on growth and seed yield of cauliflower (Brassica oleracea var. botrytis L.) in Terai zone of West Bengal. Seed Research, 30(2): 247-252.

Mishra, H. P. and Singh, B.P. 1986. Studies on nutrients and growth regulators interaction in "Snowball-6" cauliflower (Brassica oleracea var. botrytis). Progressive Horticulture, 18(1\& 2): 77-82.

Patil, A. A.; Maniur, S. M. and Nalwadi, U. G 1987. Effect GA3 and NAA on growth and yield of Cabbage. South Indian Horticulture, 35(5): 393394.

Rashid, M. M. 1999. Sabji Bitan. Text Book Division, Bangla Academy, Dhaka, Bangladesh. p. 217.

Reddy, S. A. 1989. Effect of foliar application of urea and gibberellic acid on cauliflower (Brassica oieracea var. hotrytis). Journal of Research Association of Pan African Unity, 17(1): 79-80.

Srivastava, V. 1960. Studies on crop maturity in cauliflower. Relationship between the time of curd initiation and curd maturity of plants within a cauliflower crop. Journal of Horticultural Science, 44(2): 129.

UNDP. 1998. Land Resource Appraisal of Bangladesh for Agricultural Development Report 2. Agroecological Regions of Bangladesh, FAO, Rome, Italy, pp. 577.

Vijay, K. and Ray, N. 2000. Effect of growth hormones on cauliflower. Pant subhra. Orissa Journal of Horticulture, 28(1): 65-67.

Yabuta, R. P.; Joshi, R. P.; Singh, R. D. and Adhikari, K. S. 1981. Effect of $\mathrm{GA}_{3}$ on the performance of cauliflower plants variety "Snowball-16". Progressive Horticulture, 5(1): 35-38 